\title{
Susceptibility to Type 1 (insulin-dependent) diabetes mellitus is determined by MHC class II molecules. What about DR4?
}

\author{
C.H.Mijovic, D.Jenkins, M. A.Penny \\ Department of Medicine, University of Birmingham, Birmingham, UK
}

A recent review article by Thorsby and Rønningen [1] claimed that "particular HLA-DQ molecules play a dominant role in determining susceptibility or resistance to Type 1 (insulin-dependent) diabetes mellitus". Their evidence was based on the finding that predisposition to Type 1 diabetes is consistently associated with particular combinations of DQA1 and DQB1 genes encoded either in cis or trans in three different ethnic groups: Blacks, Caucasians and Japanese. The DQA1 0301/DQB1*0401 heterodimer encoded in cis on Japanese haplotypes and the similar DQA1*0301/DQB1*0402 heterodimer encoded in trans in Blacks and Caucasians were described as important susceptibility molecules. In our study of Type 1 diabetes in the Chinese, however, the DQA1"0301/ DQB1 ${ }^{*} 0401$ heterodimer (encoded in cis or trans) occurred in a lower frequency amongst the diabetic patients ( $4.9 \%$ of 41 ) as compared to the healthy control subjects (14.5\% of 83) [2]. This finding of a contrasting disease association with the same DQ molecule in two different races has several possible explanations. 1) The DQA1 ${ }^{*} 0301 / \mathrm{DQB} 1^{*} 0401$ molecule is not a susceptibility determinant for Type 1 diabetes. 2) The DQA1"0301/ DQB1 ${ }^{*} 0401$ molecule is a susceptibility determinant but its effect is modified by another product of the Chinese genotype. This product might be MHC or non-MHC encoded. 3) A factor necessary for disease penetrance in the presence of the DQA1*0301/DQB1*0401 molecule is absent from the Chinese environment. 4) There is heterogeneity between Type 1 diabetes in the Japanese and the Chinese.

Currently, none of these possibilities can be excluded. This example is just one anomaly not addressed by a model proposing a direct involvement for particular DQ molecules in determining susceptibility to Type 1 diabetes. Below we present the evidence for and against such a model and propose a modification to it.

The evidence for a role for DQ molecules in susceptibility to Type 1 diabetes is supported by studies of the NOD mouse. The NOD mouse expresses a single distinctive class II molecule I-A ${ }^{\text {nod }}$ (I-A is the murine homologue of DQ). The second type of murine class II molecule, I-E, is not expressed due to a non-functional I-E $\alpha$ chain gene. Insulitis in NOD mice can be prevented by the introduction of I-E $\alpha$ chain or non-NOD I-A transgenes [3]. In humans a role for DQ in protection against the disease is indicated by the consistent reduction in frequency of the DQB1"0602 and/or the similar 0603 alleles in Type 1 diabetic patients of all races studied [reviewed in 4]. Similarly DR3 (DRB1*0301) and its associated DQ molecule DQA1 $10501 / \mathrm{DQB} 1 * 0201$ is increased in frequency in diabetic patients of all races except the Japanese in whom it is a rare allele. The available data are thus compatible with a role for DQ in protection from the disease and in DR3-associated susceptibility to it.

Data implicating a role for DQ in DR4-associated susceptibility are less convincing. The DQ molecule DQ8 encoded by DQA1"0301/DQB1 0302 has been implicated in DR4-associated susceptibility. A closer examination, however, shows that not all DR4 haplotypes encoding DQ8 are associated with disease. In white Caucasians only DRB1*0401 and DRB1*0402 haplotypes carrying DQ8 are positively associated with disease; DRB1 ${ }^{*} 0403,0404$ and 0405-DQ8 positive haplotypes are not [5]. In Japanese DRB1*0406-DQ8 haplotypes are reduced amongst diabetic patients as compared to control subjects whereas DRB1*0802-DQ8 haplotypes are increased [6]. A modifying effect of the various DRB1 alleles on the susceptibility encoded by the DQ8 molecule has been proposed to explain these findings in Japanese and Caucasians.

Such a modifying effect of DRB1, however, cannot explain the contrasting disease associations shown by the DQA1"0301/DQB1"0401 encoded DQ molecule in the Japanese (positive) and Chinese (negative). This molecule is encoded in cis on DRB1"0405 positive haplotypes in both races. Other differences on the DRB1"0405DQA1"0301-DQB1*0401 haplotype in the two races may thus be implicated suggesting the involvement of nonDR/DQ susceptibility factors.

The study of diabetic subjects heterozygous for DR4 haplotypes adds further complexity to the role of DQ8 in determining susceptibility to Type 1 diabetes. Data from 
white Caucasians suggest that the increase in DR4-DQ8 haplotypes amongst diabetic patients may be confined to DR3/4 heterozygotes $[7,8]$. DR1/4 heterozygosity is also associated with an increased risk for Type 1 diabetes. Amongst DR1/4 heterozygote diabetic patients the DRB1 ${ }^{*} 0401$ allele is increased as compared to DR4-positive control subjects but the DQ8-encoding DQB1*0302 allele is not [8]. DR4/X patients (where $\mathrm{X}$ is not $1,3,4$ or 5 ) also showed a distribution of DR4-associated DQ alleles similar to that seen in DR4 control subjects [7]. The data implicating DQ8 as a factor in DR4-associated susceptibility are thus not universal.

A role for trans-encoded DQ molecules in determining the synergistic effects on risk observed for DR3/4 heterozygotes has been proposed. If this is the case then a role for trans-encoded DQ molecules in other heterozygous combinations exhibiting an interactive effect on risk might be expected e.g. DRB1*0405-DQA1 ${ }^{*} 0301$ DQB1 ${ }^{*} 0401 / D R B 1{ }^{*} 0802-D Q A 1{ }^{*} 0301-D Q B 11^{*} 0302$ in the Japanese [6]. These heterozygotes, however, cannot form any additional trans-encoded species of DQ heterodimer as only the DQA1*0301 allele is present.

Mode of inheritance studies indicate that the DR3 and DR4-associated susceptibility factors are different and interact synergistically. The data are compatible with recessive inheritance of the DR3-associated factor in the absence of DR4 and dominant inheritance of the DR4-associated factor in the absence of DR3 [9]. Any hypothesis on susceptibility determinants for Type 1 diabetes must take these findings into account. Data from the NOD mouse show that the susceptibility conferred by $\mathrm{I}-\mathrm{A}^{\text {nod }}$ can be overcome by the introduction of I-E or other I-A molecules and is thus recessive. This is compatible with the possibility that DR3-associated susceptibility is DQ-encoded whereas DR4-associated susceptibility is not.

In general the literature on DQ heterodimers should be treated with caution. Counting of heterodimers is not reliable in heterozygous individuals - certain pairings do not occur and preferential formation of particular DQ heterodimers cannot be excluded [10]. It is thus currently not possible to predict accurately the DQ heterodimer status of heterozygous individuals from genotyping data alone. In DR3/4 heterozygotes, for example, there is no evidence to confirm that all four possible heterodimers are expressed. In the single structural study of the DQ heterodimers encoded by DR3/4 heterozygotes the transencoded DQA1"0501/DQB1*0302 was detected [11]. The occurrence of the other possible trans-encoded heterodimer DQA1*0301/DQB1*0201 has yet to be confirmed. This may cause a large margin of possible error in the counting of heterodimers in patients and control subjects and hence in the calculation of relative risks.

At present none of the proposed models of a mechanism for the involvement of DQ molecules in suscepti- bility to Type 1 diabetes take into account all of the available data. Whilst DQ molecules might have a role current evidence does not support simple models invoking susceptibility conferred by certain DQ heterodimers. There is good evidence for a role of DQ molecules in resistance to the disease. A role for the DQ molecule encoded by DQA1 ${ }^{*} 0501 / \mathrm{DQB} 1^{*} 0201$ in DR3-associated susceptibility cannot be excluded. DQ molecules, however, cannot be the sole contributors to the susceptibility associated with DR4. We should not be reluctant to propose models where a component of the MHC-associated susceptibility to Type 1 diabetes is neither DR-nor DQ-encoded.

\section{References}

1. Thorsby E, Rønningen KS (1993) Particular HLA-DQ molecules play a dominant role in determining susceptibility or resistance to type 1 (insulin-dependent) diabetes mellitus. Diabetologia 36: $371-377$

2. Penny MA, Jenkins D, Mijovic CH et al. (1992) Susceptibility to IDDM in a Chinese population. Role of HLA class II alleles. Diabetes 41: $914-919$

3. Lund T, O'Reilly L, Hutchings P et al. (1990) Prevention of insulin-dependent diabetes mellitus in non-obese diabetic mice by transgenes encoding modified I-A $\beta$-chain or normal I-E $\alpha$ chain. Nature 345: 727-729

4. Mijovic CH, Todd JA, Barnett AH (1991) Trans-racial gene mapping studies. Clin Endocrinol Metab 5: 321-340

5. Sheehy MJ, Scharf SJ, Rowe JR et al. (1989) A diabetes-susceptible HLA haplotype is best defined by a combination of HLADR and -DQ alleles. J Clin Invest 83: 830-835

6. Awata T, Kuzuya T, Matsuda A, Iwamoto Y, Kanazawa Y (1992) Genetic analysis of HLA class II alleles and susceptibility to type 1 (insulin-dependent) diabetes mellitus in Japanese subjects. Diabetologia 35: 419-424

7. Tait BD, Mraz G, Harrison LC (1988) Association of HLADQw3 (TA10-) with Type 1 diabetes occurs with DR3/4 but not DR1/4 patients. Diabetes 37:926-929

8. Erlich HA, Bugawan TL, Scharf S, Nepom GT, Tait B, Griffith RL (1990) HLA-DQ $\beta$ sequence polymorphism and genetic susceptibility to IDDM. Diabetes 39:96-103

9. Jenkins D, Fletcher J, Penny MA et al. (1991) DRB genotyping supports recessive inheritance of DR3-associated susceptibility to insulin-dependent diabetes mellitus. Am J Hum Genet 49: 4953

10. Kwok WW, Kovats S, Thurtle P, Nepom G (1993) HLA-DQ allelic polymorphisms constrain patterns of class II heterodimer formation. J Immunol 150: 2263-2272

11. Nepom BS, Schwarz D, Palmer JP, Nepom GT (1987) Transcomplementation of HLA genes in IDDM. HLA-DQ $\alpha$ - and $\beta$ chains produce hybrid molecules in DR3/4 heterozygotes. Diabetes 36: 114-117

Dr. C.H. Mijovic

Department of Medicine

University of Birmingham

Birmingham B152TH

UK 